\title{
Research on Influences of Retaining Wall Draining Capacity on Stability of Reservoir Bank Slope Retaining Wall
}

\author{
Yisong Zhang ${ }^{1}$, 'Liming Wu ${ }^{1}$, Zijian Wang ${ }^{2,}$ and Guojun $\mathrm{Lv}^{3}$ \\ ${ }^{1}$ School of Civil Engineering\&Architecture,Chongqing Technology and Business Institute,Chongqing \\ 400052, China \\ ${ }^{2}$ School of Civil Engineering\&Architecture,Chongqing University of \\ Science\&Technology, Chongqing 401331, China
}

${ }^{3}$ School of Civil Engineering\&Architecture,Chongqing Jiaotong University, Chongqing 400074,China

Keywords: Slope retaining wall,Draining capacity,Stability;Saturation,Displacement of retaining wall.

Abstract. Taking one typical reservoir bank slope as example, do comparative analysis on internal force and displacement of slope under different draining conditions through utilizing finite element software ABAQUS. Analysis results show that thrust of slope on retaining wall obviously increases with the lengthening of rainfall time interval without draining. Horizontal displacement also presents different levels of increasing along wall height. Finally it proposes suggestions on stability measures of retaining wall thus providing references for the design, construction and maintenance of draining capacity of reservoir bank slope retaining wall.

\section{Introduction}

Rainfall in reservoir area is a common weather phenomenon which is also one of the important incentives of many engineering accidents. Most slopes become unstable and collapse during or after rainfalls [1]. Scholars at home and abroad have done a lot of work on rainfall inducing slope to be unstable. They think that rainfall infiltration and groundwater level rising lead water content of soil mass to increase and make cohesion, internal friction angle and matrix suction decrease thus reducing the shearing strength[2]. Therefore slope drainage smooth is an important determinant for its stability. The set of drainage opening on slope retaining wall is one of the important measures for draining in low slope engineering whose drainage capacity is an important factor to guarantee slope stability. During long-term drainage process, coarse particles and miscellaneous fill materials often lead drainage capacity of drainage opening to decrease or even be blocked. Its blocking would reduce the sheering strength of rock-soil mass and lead hydraulic pressure of opening behind retaining wall [3]. Continuous increasing of hydraulic pressure results in slippage or overturn failure thus leading slope to be unstable. On the basis of it, the author does simulation comparison analysis on blocking condition of drainage opening on a typical retaining wall slope. Analysis shows that hydro-static pressure constantly increases with the increasing of blocking degree of drainage opening which is an important reason for the destruction of retaining wall.

\section{Engineering Background}

Select an ideal and typical retaining wall slope to act as engineering background in which wall back is vertical and surface of rolling earth behind retaining wall is horizontal. The top of concrete retaining wall is $2 \mathrm{~m}$ wide. Bottom of it is $4 \mathrm{~m}$ wide and it is $10 \mathrm{~m}$ in height. Three rows of drainage opening are set whose positions are respectively $1 \mathrm{~m}, 4 \mathrm{~m}$ and $7 \mathrm{~m}$ away from the bottom of retaining wall. Initial groundwater level lies at the foot of slope. Elastic modulus of rock-soil mass is 10MPa and Poisson ratio is 0.3 . Cohesion $C$ is $15 \mathrm{KPa}$ and friction angle $\varphi=30^{\circ}$ [4]. Figure1 shows the relationship between saturation and permeability coefficient of material. Rainfall time interval and relationship between its saturation and matrix suction are also seen in Figure1. 


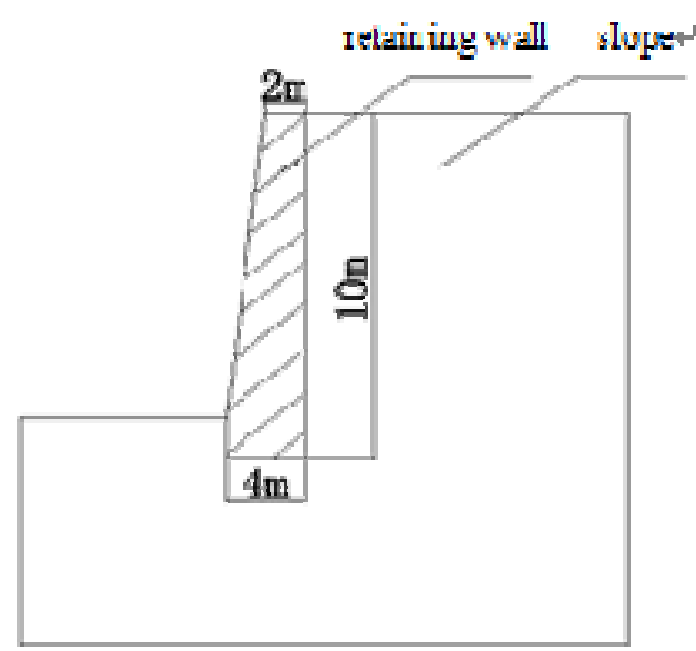

a. Diagram of Retaining Wall Slope Section

Relationship between Saturation and Permeability Coefficient

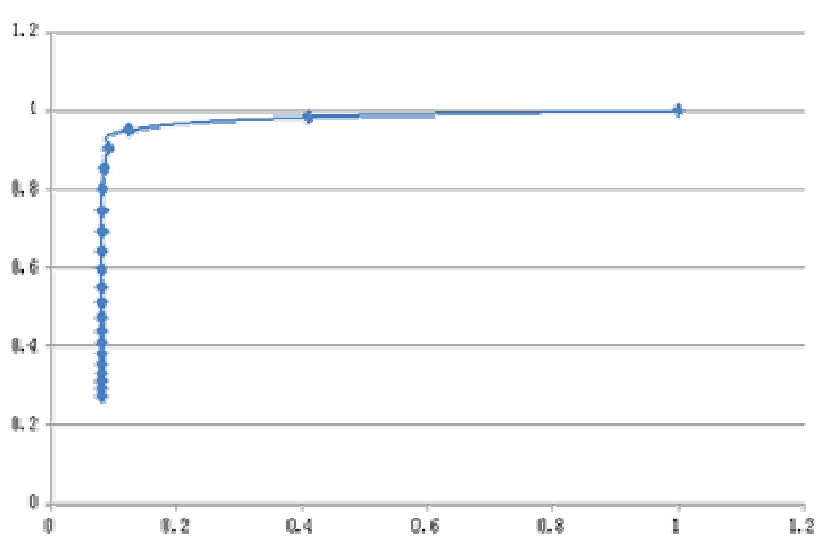

C. Diagram of Relationship between Saturation and Permeability Coefficient of Slope Rock-soil Mass

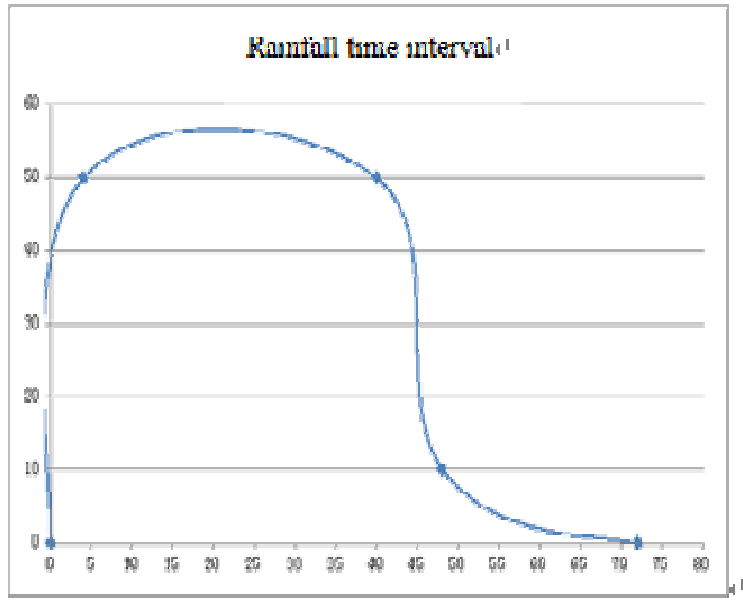

b. Curve of Rainfall Time Interva

Relationship between Saturation and Natrix Suction

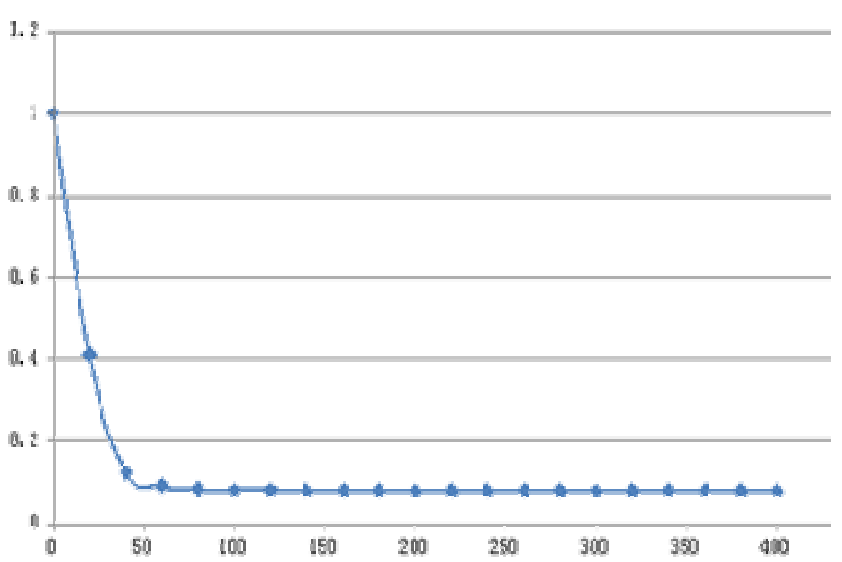

d. Diagram of Relationship between Saturation and Matrix Suction of Slope Rock-soil Mass

Figure 1. Basic Parameters of Retaining Wall and Slope

\section{Boundary Conditions of Rainfall Infiltration.}

Process of rainfall infiltration is very complex. Mein and Larson describe rainfall infiltration process and behavior utilizing three factors of rainfall intensity q,soil infiltration allowance capacity $f_{p}$ and hydraulic conductivity KWS [4].

$q<k_{w s}$ : At this moment, overland runoff does not come into being. Rainfall would totally infiltrate. Infiltration rate of water remains unchanged.

$f_{p}>q>k_{w s}$ : All rainwater infiltrates. $f_{p}$ decreases with the increasing of infiltration depth. However, rainfall intensity has not reached the soil infiltration allowance capacity. Therefore infiltration rate keeps high without decreasing. At this time, slope surface is flow boundary.

$q>f_{p}$ : As rainfall intensity is higher than infiltration capacity of soil, part of rainfall would decline after it infiltrates and becomes overland runoff capacity. By this time, soil on slope surface basically keeps in saturation situation. While rainfall reaches infiltration capacity, infiltration rate would gradually decline. Relationship between permeability coefficient and saturation and relationship between saturation and matrix suction are shown as follows:

$$
K_{w}=a_{w} K_{w s} /\left[a_{w}+b_{w} \times\left(\left(u_{a}-u_{w}\right)\right)^{c_{w}}\right]
$$


In this formula, $K_{w s}$ is the permeability coefficient when soil mass is saturated. $u_{a}$ and $u_{w}$ are atmospheric pressure and hydraulic pressure. Contact of atmosphere and slope surface is near to 0 . $a_{w}, b_{w}$ and $c_{w}$ are material coefficients.

$a S_{r}=S_{i}+\left(S_{n}-S_{i}\right) a_{s} /\left[a_{s}+\left(b_{s} \times\left(u_{a}-u_{w}\right)\right)^{c_{s}}\right]$

In the formula, $S_{r}$ is saturation. $S_{i}$ is residual saturation. $S_{n}$ is the maximum saturation whose value is $1 .{ }^{a}{ }_{s} b_{s}$ and $c_{s}$ are material coefficients.

On the basis of the above-mentioned $2^{\text {nd }}$ theory, this model establishes the time interval curve as seen in Figure1 (b) to explain the relationship between time and rainfall. This model also defines the relationship between permeability coefficient and saturation and relationship between saturation and matrix suction which are shown in Figure1 (c, d).

\section{Engineering Operating Conditions}

In order to analyze the influences of drainage capacity of different retaining wall drainage openings on stability of reservoir bank slope retaining wall, this paper utilizes comparison analysis method to do research on different operating conditions under the same rainfall time interval. Specific operating conditions are shown in Table1.

Table1. Model Analyzing Operating Conditions

\begin{tabular}{|c|c|c|c|}
\hline Operating Condition & 1 & 2 & 3 \\
\hline Name & gravity without water & $\begin{array}{c}\text { Non-drainage retaining } \\
\text { wall }\end{array}$ & $\begin{array}{c}\text { Drainage retaining } \\
\text { wall }\end{array}$ \\
\hline
\end{tabular}

\section{Stresses under Different Operating Conditions before and after Drainage}

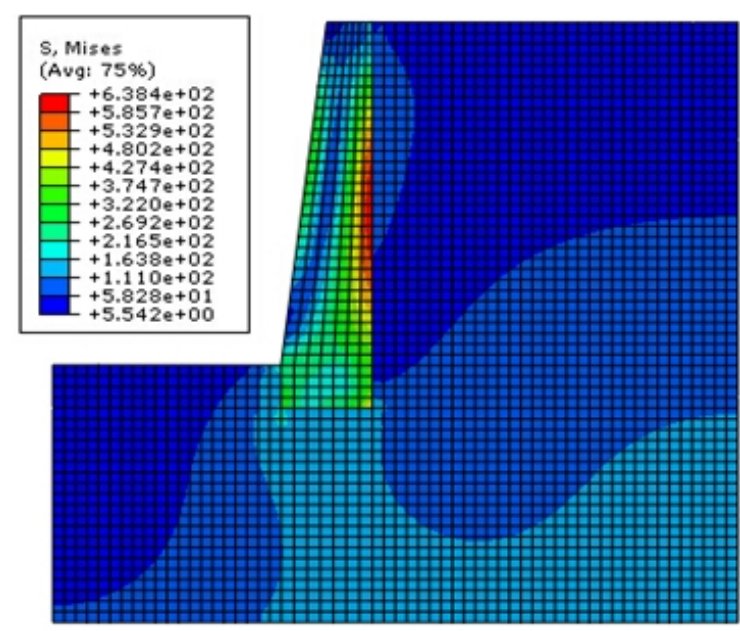

Figure2. Gravity Stress Distribution

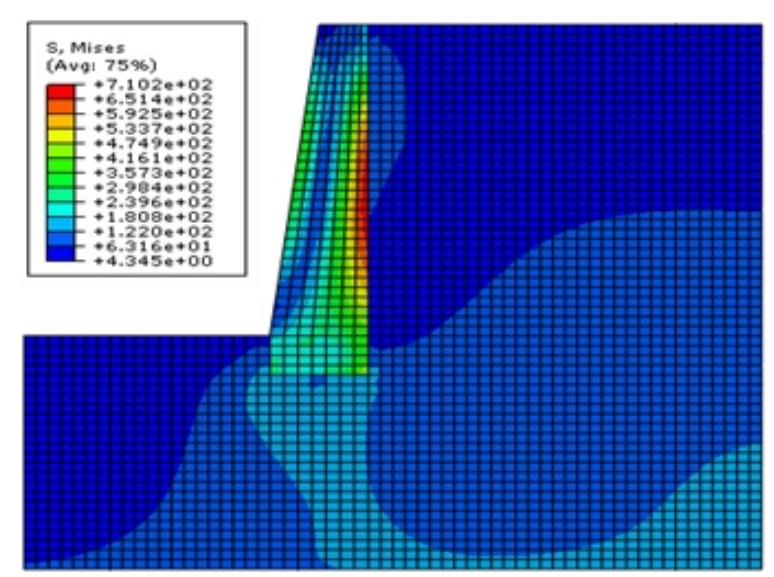

Figure4. Stress Distribution under Rainfall with Drainage

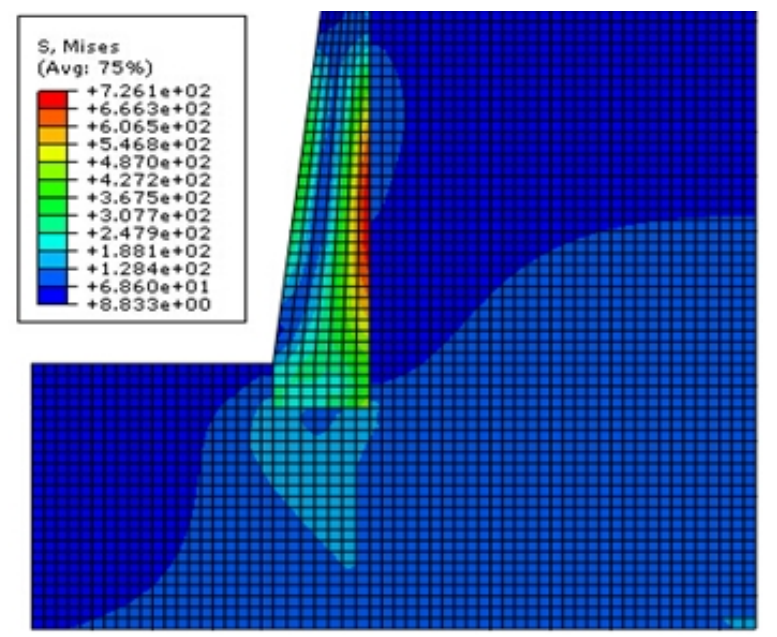

Figure3. Stress Distribution under Rainfall without Drainage

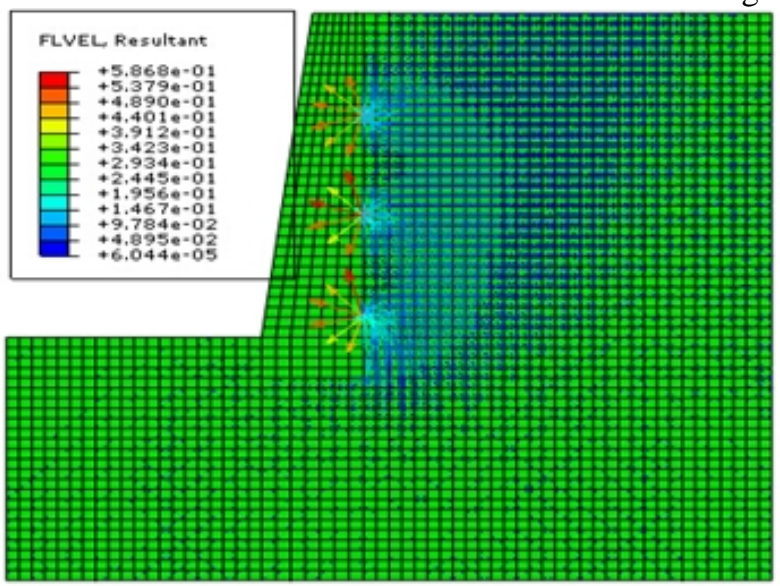

Figure5. Velocity Field under Rainfall with Drainage 

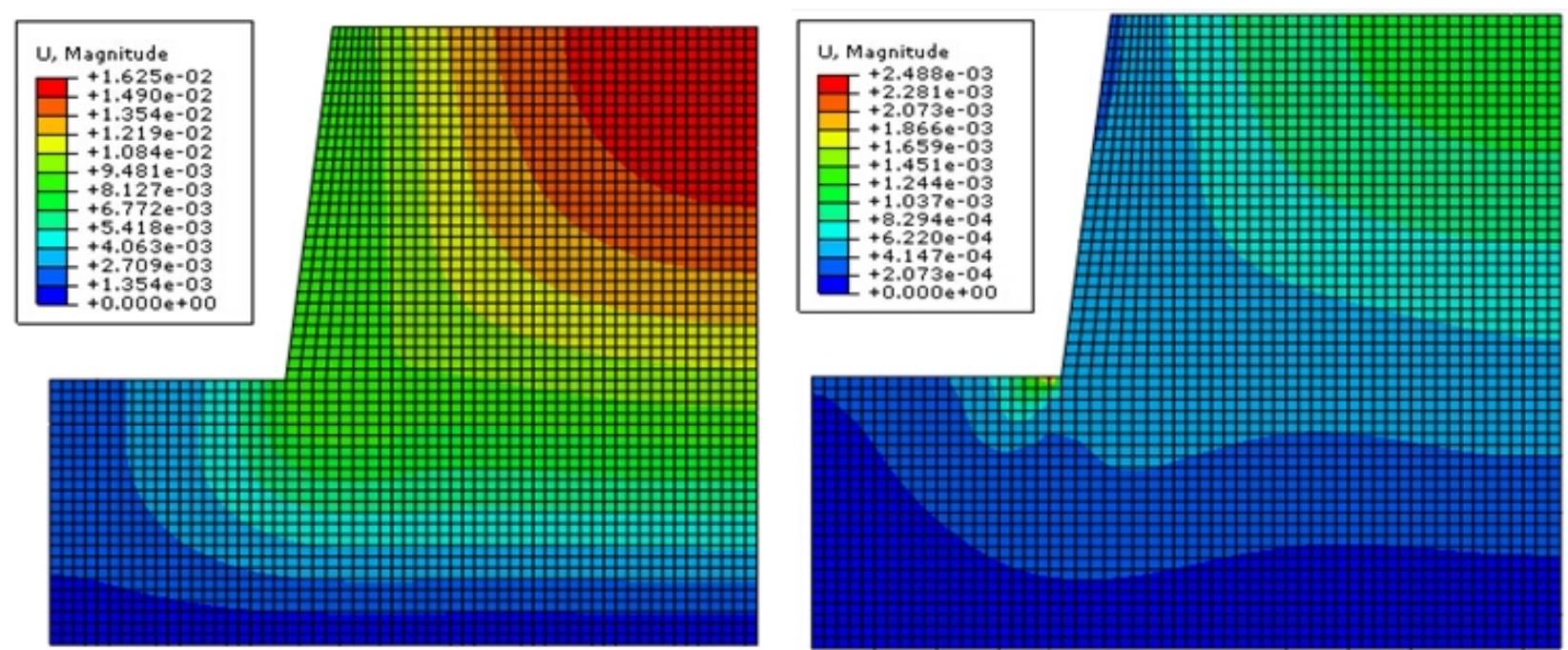

Figure6. Gravity Displacement Distribution

Figure7. Displacement Distribution under Rainfall without Drainage

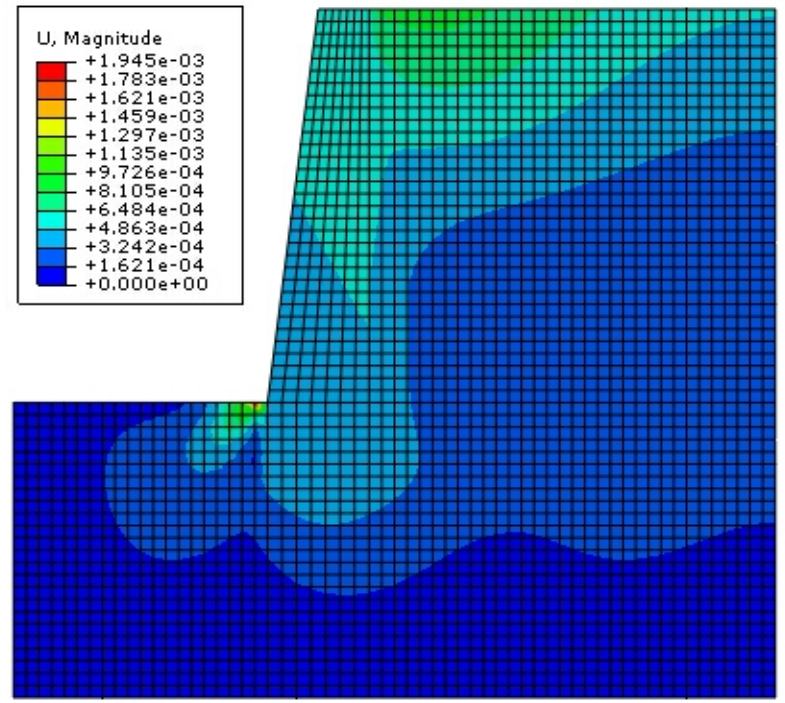

Figure8. Displacement Distribution under Rainfall with Drainage

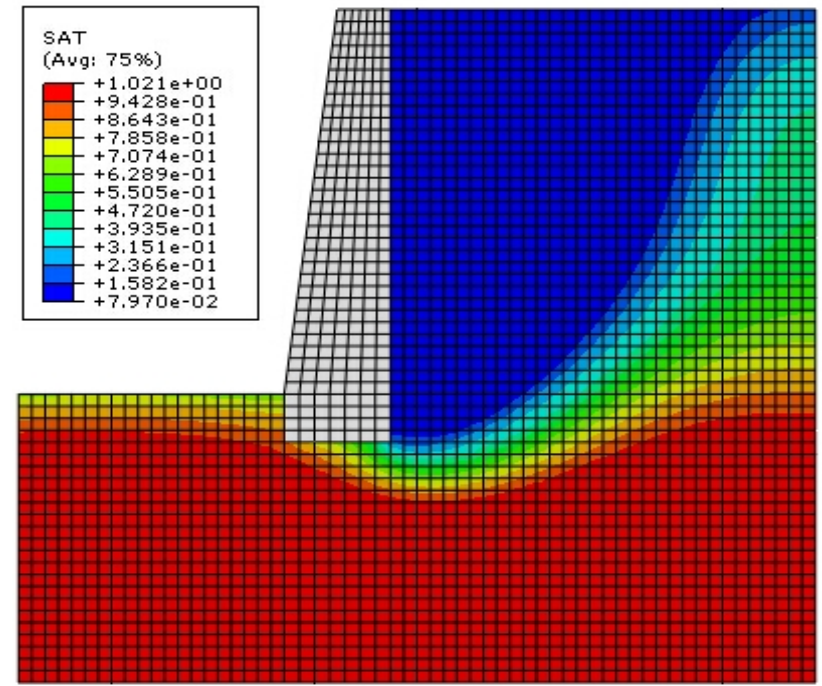

Figure9. Saturation Distribution under Rainfall with Drainage

Comparison analysis on Figure2 to Figure 5 above shows that firstly, stress in the middle part of retaining wall is high under operating conditions of gravity and being with and without drainage. Secondly, the maximum stress under rainfall without drainage is higher than that under rainfall with drainage which is also higher than that under gravity. Thirdly, stress under retaining wall base is higher than that on its left and right sides. Stress on its both sides is lower.

\section{Slope Stress and Displacement with and without Rainfall}

Comparison analysis on Figure6 to Figure8 above shows that firstly, displacement on top of retaining wall is higher under operating conditions of gravity and being with and without drainage. From top to bottom of retaining wall, displacement gradually decreases. Secondly, the maximum displacement of top point of retaining wall under rainfall without drainage is higher than that under rainfall with drainage which is also higher than that under gravity. Thirdly, influencing range of rock-soil mass deformation behind retaining wall gradually reduces from the top to the bottom of it.

\section{Curves of Stress Changing with Rainfall Time Interval under Drainage and Non-drainage Operating Conditions}

It is seen from the figure 10 that firstly, Stresses under drainage and non-drainage conditions stay the same during the first 25 hours of rainfall because soil mass keeps unsaturated. Secondly, their 
difference becomes greater and greater after 25 hours of rainfall because soil mass becomes saturated in which it is drainage openings behind the wall to take the residual rainwater away.

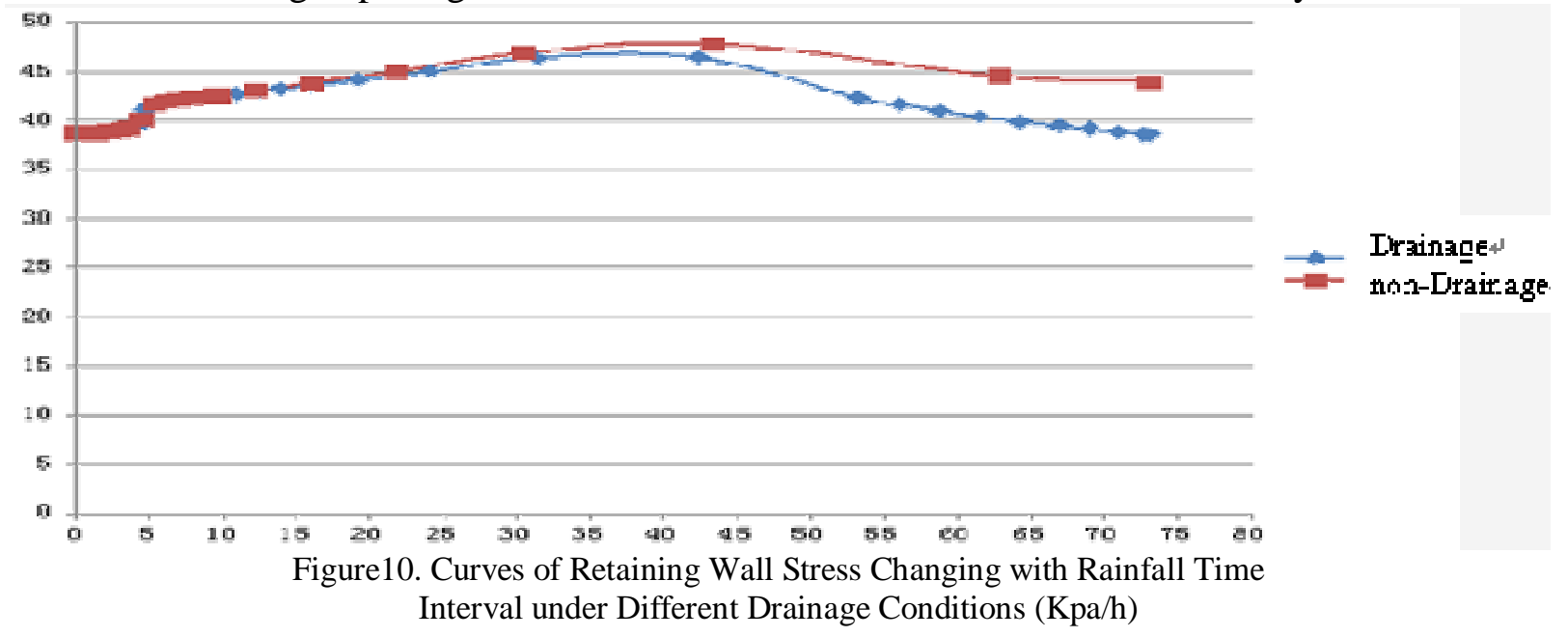

\section{Conclusions and Suggestions}

Taking a typical reservoir bank slope as example, this paper utilizes finite element software ABAQUS to do comparative analysis on internal force and displacement of slope under different drainage conditions. Through analysis, we acquire the following conclusions and suggestions.

Stress in the middle part of retaining wall is high under operating conditions of gravity and being with and without drainage. Displacement on top of retaining wall is higher under operating conditions of gravity and being with and without drainage, which gradually reduces from the top to the bottom of it.

The maximum stress and displacement of retaining wall under rainfall without drainage are higher than those under rainfall with drainage which are also higher than those under gravity.

Stress under retaining wall base is higher than that on its left and right sides. Stress on its both sides is lower.

Stresses of retaining wall under drainage and non-drainage conditions stay the same during the first 25 hours of rainfall because soil mass keeps unsaturated.

Difference of Stresses under drainage and non-drainage conditions becomes greater and greater after 25 hours of rainfall because soil mass becomes saturated in which it is drainage openings behind the wall to take the residual rainwater away.

It is suggested that influences of blocking degrees of different drainage openings, slope shapes and different physical parameters of rock-soil mass should be taken into account in the research on influences of drainage conditions on stability of retaining wall so as to lead its structure to be more reliable. Necessary confirmatory experiment research could be done under expenditure and experiment conditions being allowed.

\section{References}

[1] Yin Jun. Influence of rainfall on soil slope stability [D]. Nanjing: Nanjing University of Technology master's degree thesis, 2012.

[2] Tang Yi Qun, she Tianyu, Zhang Xiaohui et. Shear strength characteristics along with the change of water content relationship study [J]. Journal of engineering geology, 2009, 17 (2): 249-252.

[3] Zhang Hanshen, Jia Xianzhong, He Chunkai. The instability of gravity retaining wall and the strengthening of $[\mathrm{J}]$. exchange platform, 2007.72-74.

[4] Zhang Jianwei, Fei Kang. ABAQUS application in geotechnical engineering [M]. Beijing: China Water Conservancy and Hydropower Press, 2010. 